times been done, they may be mounted in a frame which has an angle where the two parts come together, so that each may be given just its proper direction.

But perhaps the most important practical lesson to be learned, from a study of the effects of obliquity of the correcting lens, is an illustration of the necessity for accuracy in the determination and correction of ametropia particularly of myopia. To place before an eye a lens that does not accurately meet its needs, may by inducing the patient to look obliquely through it, and so to encounter the cylindrical effect, prove quite disastrous for the patient.

Chas. J., æt. 2 I, came to me recently with evidences of eye-strain, headache, pain in the eyes, choroidal congestion and commencing conus. He was wearing right and left -4 . D spherical that I had ordered for him four years ago and which had been worn most of that time with comfort. But he now believed he had some astigmatism; saw with different degrees of distinctness, lines running in different directions. To see clearly objects a little way from him, he would twist his head to one side and look ob. liquely through his glasses. This maneuver gave him $\mathrm{V}$. $=\frac{15}{\mathrm{XV}}$ mostly; while looking directly forward he saw but $\frac{15}{\mathrm{Xx}}$ partly. Under a mydriatic his symptoms of eye strain promptly disappeared; it was found that during the four years his myopia had increased $0.75 \mathrm{D}$. in each eye. Such an increase may have been due to a strictly physiological development of the globe; but the effort to see clearly with his imperfect correction caused all the symptoms of an aggravated astigmatism. 'The obliquely placed lens caused an astigmatism of the incident pencil which varied continually and allowed the establishment of no constant compensatory accommodative effort.

I fear that many ophthalmologists regard it as a small matter to leave a little myopia uncorrected, or think it rather a good thing to do, because it saves a little effort of accommodation. I believe that for reasons above indicated it is dangerous; and could detain you longer with the citation of cases that would illustrate and enforce my point. As spectacle lenses are usually fitted and mounted, it is quite possible to get by looking through the edge of the lens an obliquity of the incident pencil either upward or downward of $25^{\circ}$ or $30^{\circ}$, and to the right or left of from $30^{\circ}$ to $40^{\circ}$. Now a glance at the table will in. dicate how much increase of spherical refraction accompanied by astigmatism can be obtained, by such an obliquity, with a strong lens. And if, as many, es. pecially Thomson and Green, have argued, astigmatism be especially dangerous to the myope; it cannot be doubted but that the temptation to thus secure better distant vision is also very dangerous.

The myope with a partial correction shares this danger, with the presbyope whose glass is insufficeint for near work and who strives to shorten its focusby looking through it obliquely; and the two constitute the best and most common examples of the dangers of using lenses not wisely selected.

Another very practical application of our knowledge in this direction, is in warning patients, especially such as use strong lenses, always to look through the center of the glass. And in this law governing the action of lenses we may find a partial explanation of that difficulty which Landolt so graphically portrays, in satisfying our cataract patients with the optical substitute for the crystalline lens.

\section{PATHOLOGICAL CONDITIONS OF THE TEETH AND THEIR SYSTEMIC EFFECTS.}

Read before the Section of Dental and Oral Surgery, at the Thirty.Eighth Annual Meeting of the American Medical Association, Fune, 1887 .

BY K. B. DAVIS, M.D., OF SPRINGFIELD, ILL.

This subject is one of great importance, not only to the dentist but to the oculist and the general practitioner of medicine. Important as it is, however, it has not heretofore received that attention from medical writers, and practitioners that it justly merits. That pathological condiions of the teeth and the structures adjacent to them in the oral cavity, do exert a most pernicious influence upon the general system, is a fact as well established as any other medical observation. Yet I fear there are too many practitioners that almost wholly ignore these conditions as causes of many systemic disturbances.

The attention of the profession was recently called to this subject, by the New York Medical Record, in which Dr. Samuel Sexton, a distinguished oculist and aurist of New York City, and the editor of the Record, were the principal writers.

The Medical Record of October 4, 1884, contains a report from the aural service of Dr. Sexton, entitled "Pain in the Ears, due to Irritation in the Jaws." He describes a number of cases of otalgia, in which he found the lesion to be in diseased teeth.

The same number of the Medical Recor $a$ contains an editorial on "Dead Teeth in the Jaws," which reads as follows: "Perhaps the time is near at hand when medical men should be themselves better informed concerning diseases of the jaws and mouth, rather than refer the ailments of this region to individuals whose limited knowledge of medicine does not prevent them from "treating" dead teeth long after their presence in the jaws has given rise to alveolar abscesses and neuralgias, more or less painful. It would not be strange if in the course of events, the day would soon come when all teeth without pulps, and hence, in process of more or less rapid decay, as well as those which the deposit of tarter or other cause, had entirely divested of periosteal nourishment, would be promptly condemned as unfit to remain in the jaws-regarded in fact as foreign bodies, liable to give rise, not only to cerebral irritation and diseases in the organs of special sense, through the propagation of local disturbances in the mouth, to the regions mentioned, but to endanger likewise the general health, through purulent matter discharged into the mouth from alveolar abscesses, to be continuously swallowed for a long time, or, indeed, in some instances, to be absorbed, and thus produce septicæmic poisoning. It is certainly grati- 
fying to note the establishment of instruction in oral surgery in some of the schools, and it is to be hoped that this subject will receive the attention its importance demands."

Considering the peculiar structure of the teeth and their relation through the nervous system to the general organization, the close connection existing between the teeth, the rapidity with which the flash of sympathetic pain darts along the nervous cords which vitalizes them, the intolerable and protracted suffering which ensues, upon even trifling irritation of these sensitive filaments, and remember that pain itself is fully capable of deranging the whole economy, and inducing serious and fatal disorders, we might, without the aid of much reflection, adopt the very rational conclusion that the diseases of the teeth must be of considerable consequence to the entire organization. We must also remember that the teeth were not made merely for ornament, but to perform certain specific functions, chief of which is mastication and insalivation, and that these are something more than mere forms of introduction to the stomach, that they are important to digestion, which is important to the entireness of organs, and the performance of function, and that if mastication and the insalivation accompanying it be imperfectly performed, some corresponding imperfection of digestion must result. We also know the consequences of long continued morbid influences, however unimportant in their immediate action, that disturbance of digestion constantly repeated must sooner or later develop evils of a most serious character.

The teeth are not as some seem to suppose, isolated organs, but they have an intimate and vital connection with the whole nervous system, and when the extremities of the nerves are exposed to irritation, whether by wounds of flesh or by decay of the teeth, there is a reaction upon the nervous centres, and various morbid conditions are a necessary consequence.

Mastication is doubtless the primary function of the teeth, and should these organs be unable from decay or other morbid causes, to perform this office, the stomach is called upon to perform a double function, that of mastication and digestion; and while thus compelled to do its own work and that of the teeth also, it is much more liable to become disordered, and consequently the whole system may become deranged. A stomach treated in this way for a considerable length of time must inevitably suffer. But it is possible that some other organ may first indicate functional disturbance. But should indigestion ensue from the effects of a morbid condition of the oral cavity, this deranges and impairs the whole system of nutrition and innervation, and sets to work a horrid train of morbid conditions. that render even existence itself almost intolerable.

All of us, doubtless, are frequently called upon to witness the mouths of patients whose teeth are nearly all decayed, and many of them afflicted with alveolar abscesses, the gums inflamed and receding, extremely irritable and painful, the glands of the mouth disordered, the breath fetid, the saliva cor- rupt and extremely unhealthy; the teeth are in such a morbid condition that they cannot masticate their food, but mumble it in their mouths, where it mingles with that corrupt and vicious saliva, and with all the fetid, putrescent accumulations in the cavities of those stumps of teeth, and in this condition it passes into the stomach, where it mingles with the gastric juice, tending to vitiate the whole process of digestion.

There are many other morbid conditions either developed or produced by dental irritation. Mr. Salter, in his Pathology and Surgery, in treating upon this subject, says: "The affections of the nervous system dependent on the teeth, divide themselves into those which are reflex, secondary and remote, and those which are direct, immediate and from contiguity. In the former category would rank epilepsy, neuralgia, paralysis; in the latter, local pain, facial palsy, some forms of amaurosis, etc. In other instances, such as the exalted sensibility of the tegumentary nerves of the face, erratic pains through the maxillary nerves, associated with tooth-ache, it might be difficult to say whether the phenomena are mostly reflex or direct; they probably comprise both conditions."

The situation of the teeth, and their abundant supply of nerves, and the great and diffuse swelling which their diseases produce in contiguous structures, inevitably involve much nervous disturbance and complication. The inferior molars are but little removed from the tonsils, and Eustachian tube, from the parotid region and the external auditory passage. The fangs of the superior molars are close to the orbit, and its all important contents; and more posteriorly they approach the spheno-maxillary fossa and fissure. Thus is it easy to account for the nervous complications which are directly entailed by the spread of inflammation from the periosteum of diseased teeth.

The several branches of the trigeminus appear to be the most susceptible of reflex affection, caused by dental irritation of one of them; but next to the different elements of the fifth nerve, the branches of the cervical and brachial plexuses are the most commonly involved-pains of the neck, shoulder, acromian process, insertion of the deltoid, bend of the elbow, are by no means uncommon, and with them occasionally a loss of motor power, a weary sense of fatigue in the flexor muscles, and an inability to grasp firmly with the hand.

Pain is only one of the phenomena of reflex dental nerve irritation. It may induce muscular spasm, muscular paralysis, paralysis of some of the nerves of special sense, perverted nutrition.

As regards the teeth themselves, which excite this exalted nervous irritability, nearly all their diseases appear capable of causing this condition.

Caries with or without exposure of the pulp exostosis, hypertrophy of the cementum, nodular developments of dentine in the pulp cavity, periostitis, plastic or suppurative, impaction of permanent teeth in the maxillary bone, crowding of teeth for want of room.

Each and all of the above mentioned pathological conditions of the teeth may and have caused mani- 
festations of reflex nervous irritation, though they may exist in a severe form without producing any such result.

Of the nervous and complicated morbid conditions produced from dental diseases, the following may be mentioned as occurring less frequently than those above mentioned : Chronic trismus, epilepsy, tetanus, partial paralysis, amaurosis, deafness, opthalmia, and perverted nutrition.

It is not deemed necessary to occupy your time in the narration of cases illustrating the foregoing observations, but I will refer you to the works of Salter, Bond, Garrison, and our periodical literature, where they may be found and read at your leisure.

From the foregoing observations, it is surely very important that the physician should never disregard the dental orgaus as agencies in the production or continuance of diseases; and it should also deeply impress the dental practitioner with the importance of a clear and comprehensive knowledge of the entire organism, both in health and in a diseased condition. He should ever bear in mind that his operations are not upon isolated organs merely, but upon organs bearing very intimate and close sympathetic relations to the entire economy.

PISTOL-SHOT WOUND OF THE ABDOMEN; LAPAROTOMY AND SUTURE OF THE WOUNDED INTESTINES.

Read before the Medical Society of Virginia, October 22, 1887 , BY A. S. PRIDDY, M.D., of KEYSVILLE, VA.

Ras Wimbish, a negro, aged 60 years, was twice shot by his employer, with whom he was engaged in an altercation, on September 4, I887, about 8 P.M., with a Smith \& Wesson revolver, $3 z$ calibre, at a distance of six or eight feet. The shooting occurred on a farm in Mecklenburg county, about four miles from the town of Chase City. The wounded man, at the last discharge of the pistol, exclaimed that he was shot, ran to the stables, mounted a horse, and rode into town where his family lived, "to get a doctor to cut the balls out." Dr. C. W. Walker was called in, and found him falling into a state of profound shock, from which it was several hours before he began to rally. He was, after recovering from the shock, very restless, with an anxious countenance, much thirst, pulse quick and weak, and hiccoughed slightly, though vomited none. Dr. Walker administered $1 / 3$ grain of morphia, and proceeded to examine him.

He found that the first shot had entered the fleshy portion of the thigh, and passing through was lodged under the skin on posterior aspect, from which it was easily extracted. The second shot had taken effect about one-half inch below the anterior superior spine of the ilium, toward the abdomen, the point of entry being small, presenting a jagged appearance, and was sealed with a clot of blood. Abdominal palpation revealed nothing wrong, except considerable pain on pressure.
He complained of an excruciating pain in abdomen. The track of the ball was not fully explored with a metallic probe, as it was quite certain, from its direction, that it had entered the abdominal cavity. The man was put to bed and the usual "ostrich plan" of treatment pursued; that is, warm applications to the bowels, and sufficient opium to control pain, to await developments in the case.

On the next morning the patient was found to have passed a fairly comfortable night, under opium. He had voided a large quantity of clear urine, but his bowels had not moved since the shooting. The abdominal pain and tenderness had increased. The abdomen had become very tympanitic and emphysematous, which symptoms were attributed to a gaseous distension of the abdominal walls, or of the intestines. This condition was of such importarce as to make death seem imminent from dyspnœa.

Thus things continued: dyspnœa, hiccough, occasional retching and vomiting, slightly elevated $t \in m$ perature, and an obstinate constipation, till the third day after the shooting, when a cathartic was given wnich in due time produced several evacuations, containing considerable blood. This made intes. tinal perforation certain beyond cavil. After this the tympanitic condition of the abdomen disappeared considerably for nearly twenty-four hours, after which the before-mentioned condition again became just as alarming, and so continued.

On September 8th, the fourth day after the shooting, Dr. F. J. Gregory, of this place, and I were telegraphed for. We arrived about 9 o'clock that night, and found the man in the condition before mentioned; and according to Dr. Walker he was growing weaker. There were also slight peritonitic symptoms. We advised an exploratory incision, with several objects in view: first, to relieve the gaseous distension, which was fast causing his death from dyspncea; to cleanse the cavity of all extravasations and to repair damage to the wounded viscera; and give free drainage, which might lessen his chances of death from suppurative peritonitis, septicæmia, etc., or at least make his time of living more comfortable, and his death one not attended with so much suffering. These views were concurred in by Dr. Walker, though we thought that the "golden opportunity" had been lost by waiting so long. Yet, at earnest solicitation of the wounded man to give him every known chance for his life, however slight that chance might be, we decided on a laparotomy the next morning, provided the man were living.

The operation was performed on September $g^{\text {th, }}$ the patient being still alive. I made preparations for the operation, which were about as complete as could be made in a negro cabin, but not so elegant as some of our well equipped city hospitals afford. The instruments were placed in a 5 per cent. solution of carbolic acid, and the sponges thoroughly washed out in that solution. The hands and nails of all the participants in the operation were cleansed with soap and water, and then dipped in carbolized oil. The toilet of the abdomen was made by scouring the walls, shaving the pubes, and washing the whole over with a 5 per cent. solution of carbolic 